\title{
The Interaction between Logic and Geometry in Aristotelian Diagrams
}

\author{
Lorenz Demey ${ }^{1}$ and Hans Smessaert ${ }^{2}$ \\ 1 Center for Logic and Analytic Philosophy, KU Leuven \\ Lorenz. Demey@hiw . kuleuven . be \\ 2 Department of Linguistics, KU Leuven \\ Hans.Smessaert@arts.kuleuven. be
}

\begin{abstract}
We develop a systematic approach for dealing with informationally equivalent Aristotelian diagrams, based on the interaction between the logical properties of the visualized information and the geometrical properties of the concrete polygon/polyhedron. To illustrate the account's fruitfulness, we apply it to all Aristotelian families of 4-formula fragments that are closed under negation (comparing square and rectangle) and to all Aristotelian families of 6 -formula fragments that are closed under negation (comparing hexagon and octahedron).
\end{abstract}

Keywords: Aristotelian diagram, logical geometry, square of oppositions, hexagon, octahedron, cross-polytope, symmetry group.

\section{Introduction}

Aristotelian diagrams are compact visual representations of the elements of some logical or conceptual field, and the logical relations holding between them. These diagrams have a long and rich history in philosophical logic [26]. Today, they are still widely used in logic 1124, but also in fields such as cognitive science, linguistics, philosophy, neuroscience, law and computer science [17/19/21] (see [12, Section 1] for more examples). Aristotelian diagrams have thus come to serve "as a kind of lingua franca" [20, p. 81] for a highly interdisciplinary community of researchers concerned with logical reasoning. Logical geometry $\sqrt{1}$ systematically investigates Aristotelian diagrams as objects of independent interest (regardless of their role as lingua franca), exploring various abstract-logical topics [12[14]15/33] as well as some more visual-geometrical issues [10[16 31|32].

One of the major visual-geometrical issues studied in logical geometry is the fact that a single logical structure often gives rise to a wide variety of different visualizations. In other words, even after all the strictly logical 'parameters' of a structure have been fixed, one is still confronted with several design choices when drawing the actual Aristotelian diagram for that structure. This phenomenon is widely manifested in the extant literature: there are numerous cases of authors who use completely different Aristotelian diagrams to visualize one and the same

\footnotetext{
${ }^{1}$ See www . logicalgeometry . org
} 
underlying logical structure (concrete examples will be provided below). Furthermore, since authors typically use Aristotelian diagrams to help their readers gain a better insight into some underlying logical structure, the fact that a single structure can be visualized by means of different diagrams naturally raises the question whether some of these diagrams are perhaps more 'effective' (i.e. have a greater positive impact on readers' comprehension) than others.

This issue has already been partially addressed in other work. For example, 13. compares different Aristotelian diagrams (a hexagon and an octhaedron) for the Boolean algebra $\mathbb{B}_{3}$, and examines their geometrical connections with the Hasse diagram for $\mathbb{B}_{3}$. Similarly, [35] compares two Aristotelian diagrams (a rhombic dodecahedron and a nested tetrahedron) for the Boolean algebra $\mathbb{B}_{4}$. These existing studies have a number of limitations, however: on the logical side, they are restricted to structures that are Boolean closed (i.e. that constitute entire Boolean algebras), and on the geometrical side, they are restricted to comparing Aristotelian diagrams that are based on different geometric shapes.

The main aim of the present paper is therefore to propose and illustrate a new approach to systematically investigate different Aristotelian diagrams for a given underlying logical structure. We will show that this approach does not suffer from the limitations present in other work: logically speaking, it applies to structures that are Boolean closed as well as to structures that are not Boolean closed, and geometrically speaking, it applies to Aristotelian diagrams that are based on different geometric shapes as well as to Aristotelian diagrams that are based on the same geometric shape. The key idea of the new approach is that for any given set of logical formulas $\mathcal{F}$, one can calculate a number $\ell(\mathcal{F})$ based on strictly logical considerations; similarly, for any concrete Aristotelian diagram $\mathcal{P}_{\mathcal{F}}$ that visualizes $\mathcal{F}$, one can calculate a number $g\left(\mathcal{P}_{\mathcal{F}}\right)$ based on strictly geometrical properties. The interaction between $\ell(\mathcal{F})$ and $g\left(\mathcal{P}_{\mathcal{F}}\right)$ will turn out to be very informative about the quality of $\mathcal{P}_{\mathcal{F}}$ as a visualization of $\mathcal{F}$.

The paper is organized as follows. Section 2 introduces some basic notions from logical geometry, and explains the distinction between informational and computational equivalence of Aristotelian diagrams. Section 3 then discusses the interaction between logical and geometrical properties of Aristotelian diagrams on a wholly general level. Next, Sections 4 and 5 investigate the concrete details of this logico-geometrical interaction in Aristotelian diagrams with 4 and 6 formulas, respectively. Finally, Section 6 summarizes the results obtained in this paper, and discusses the advantages as well as the limitations of the logicogeometrical perspective.

\section{Informational and Computational Equivalence}

Given a logical system $\mathbf{S}$ and a set $\mathcal{F}$ of formulas from that system, an Aristotelian diagram for $\mathcal{F}$ in $S$ is a diagram in which the formulas of $\mathcal{F}$ and the Aristotelian relations holding between those formulas are visualized by means of

points and lines connecting those points, respectively. The Aristotelian relations are defined as follows: two formulas $\varphi$ and $\psi$ are said to be 
(a)

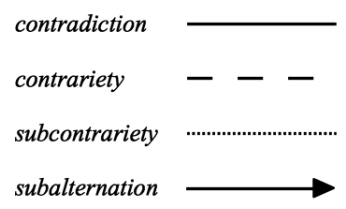

(b)

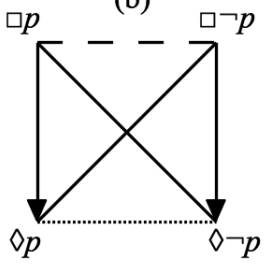

(c)

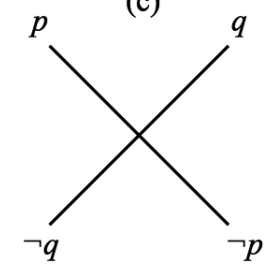

Fig. 1. (a) Visual code for the Aristotelian relations, (b) classical square for formulas from the modal logic S5 ( $\square$ and $\diamond$ should be read as 'necessarily' and 'possibly', respectively), (c) degenerated square for formulas from propositional logic.

$\begin{array}{lllll}\text { S-contradictory } & \text { iff } & \mathrm{S} \models \neg(\varphi \wedge \psi) & \text { and } & \mathrm{S} \models \varphi \vee \psi, \\ \text { S-contrary } & \text { iff } & \mathrm{S} \models \neg(\varphi \wedge \psi) & \text { and } & \mathrm{S} \not \models \varphi \vee \psi, \\ \text { S-subcontrary } & \text { iff } & \mathrm{S} \not \models \neg(\varphi \wedge \psi) & \text { and } & \mathrm{S} \models \varphi \vee \psi, \\ \text { in S-subalternation } & \text { iff } & \mathrm{S} \models \varphi \rightarrow \psi & \text { and } & \mathrm{S} \not \models \psi \rightarrow \varphi .\end{array}$

Informally, the first three relations are concerned with whether the formulas can be true/false together, whereas the fourth relation is concerned with truth propagation [33. These relations will be visualized using the code shown in Fig. 1(a). Finally, two formulas are said to be unconnected iff they do not stand in any Aristotelian relation at all.

The contemporary literature on Aristotelian diagrams has mainly focused on the logical aspects of these diagrams. This is clearly manifested in the classification of Aristotelian diagrams into different families. For example, with respect to 4-formula-diagrams, we distinguish between the 'classical square of oppositions' and the 'degenerated square', as shown in Fig. 1 (b) and (c), respectively. Similarly, with respect to 6-formula-diagrams, we distinguish between the 'JacobySesmat-Blanché (JSB) hexagon', the 'Sherwood-Cżezowski (SC) hexagon' and the 'U4 hexagon' (among others), as shown in Fig. 2(a), (b) and (c), respectively ${ }^{2}$ The differences between these families of Aristotelian diagrams are all based on their logical properties. First of all, different families often have different Aristotelian relations; e.g. the classical square does not contain pairs of unconnected formulas, whereas the degenerated square contains 4 unconnected pairs. Secondly, different families may have different constellations of Aristotelian relations; e.g. the JSB hexagon and the SC hexagon both have 3 contrarieties, but in the former they constitute a closed triangle, whereas in the latter they do not [31. Thirdly, different families often have a different Boolean structure; e.g. the Boolean closure of the classical square is (isomorphic to) $\mathbb{B}_{3}$, whereas the Boolean closure of the degenerated square is (isomorphic to) $\mathbb{B}_{4}[14$.

Using terminology from Larkin and Simon 23, the Aristotelian diagrams in Figs. $1(\mathrm{~b}-\mathrm{c})$ and $2(\mathrm{a}-\mathrm{c})$ are not informationally equivalent. They visualize different logical structures - i.e. different chunks of logical information-, and

\footnotetext{
${ }^{2}$ See [15] for some historical background on this nomenclature.
} 


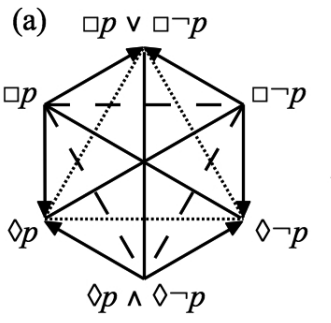

(b)

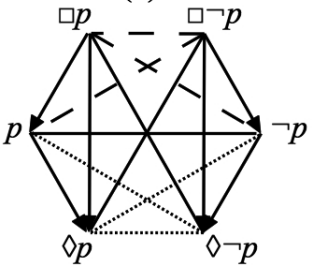

(c) $\quad p \vee \square \neg p$

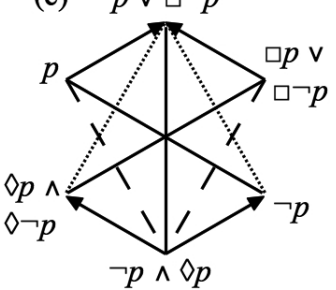

Fig. 2. (a) JSB hexagon, (b) SC hexagon and (c) U4 hexagon for formulas from S5.
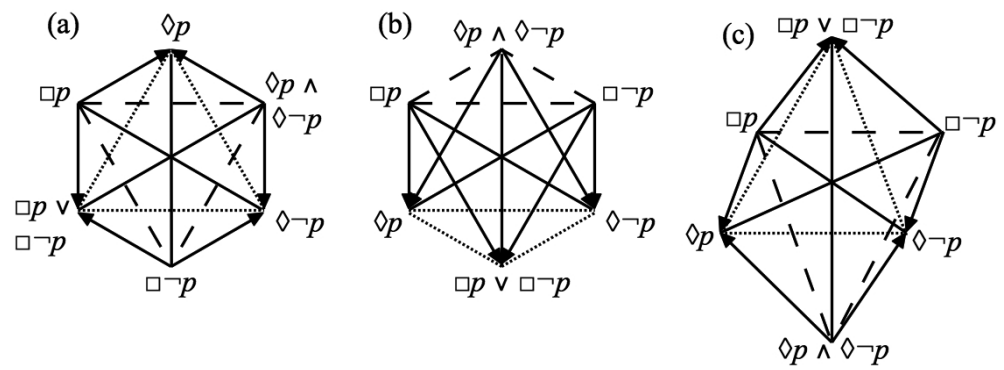

Fig. 3. Three visual alternatives to the JSB hexagon in Fig. 2(a).

hence, the differences between these diagrams are entirely due to differences between their underlying logical structures.

A completely different type of question arises when we decide to focus on a single logical structure (i.e. one set of formulas in one logical system), and investigate the various Aristotelian diagrams that have been used to visualize this single structure. For example, given four formulas that constitute a classical square, this square is usually drawn as in Fig. 1(b), but it has also been drawn with the subalternations pointing upwards, from left to right, etc. [20. Similarly, given six formulas that constitute a JSB hexagon, this hexagon is usually drawn as in Fig. 2(a), but it has also been drawn as shown in Fig. 3(a) 29] or Fig. 3.(b) [5]25]. This structure has also been visualized by means of a different geometric shape altogether, viz. an octahedron, as shown in Fig. 3(c) 2230 .

Again using terminology from Larkin and Simon [23], the Aristotelian diagrams in Figs. 2(a) and $3(\mathrm{a}-\mathrm{c})$ are informationally equivalent (they are different visualizations of one and the same logical structure), but they are not computationally equivalent. After all, even though the visual differences between these diagrams are irrelevant from a strictly logical perspective, they can significantly influence the diagrams' effectiveness in increasing user comprehension.

\section{Logic versus Geometry in Aristotelian Diagrams}

We will now present a general approach to study the interaction between logical and geometrical properties of informationally equivalent Aristotelian diagrams. 
We will focus exclusively on fragments $\mathcal{F}$ that are closed under negation, i.e. if $\varphi \in \mathcal{F}$, then also $\neg \varphi \in \mathcal{F}$ (up to logical equivalence). Such fragments always have an even number of formulas, and it will be fruitful to view them not only as consisting of $2 n$ formulas, but also as $n$ "pairs of contradictory formulas" (PCDs). Additionally, we will only deal with Aristotelian diagrams in which negation is visually represented by means of central symmetry, so that $\varphi$ and $\neg \varphi$ correspond to diametrically opposed points in the diagram. It should be emphasized that both the logical condition (closed under negation) and the geometrical condition (central symmetry) are satisfied in nearly every Aristotelian diagram that has ever been produced ${ }^{3}$ and are thus very mild restrictions.

We know from basic combinatorics that a fragment of $2 n$ formulas (i.e. $n$ PCDs) can be ordered in exactly $(2 n)$ ! ways ${ }^{4}$ However, this number does not take into account the fragment's PCD-structure, in the sense that a formula and its negation are not treated any differently from any other pair of formulas. If we only consider orderings that respect the fragment's PCD-structure, we find the number $2^{n} \times n$ !. On the one hand, there are $n$ PCDs to be ordered, which yields the second factor $(n !)$; on the other hand, each of these $n$ PCDs has 2 'orientations', viz. $(\varphi, \neg \varphi)$ and $(\neg \varphi, \varphi)$, thus yielding the first factor $\left(2^{n}\right)$. Note that this formula is strictly based on the logical properties of the fragment, viz. the facts that it contains $2 n$ formulas and is closed under negation.

A fragment of $n$ PCDs can be visualized by means of a polygon or polyhedron that has $2 n$ vertices and is centrally symmetric (so that the diagram's vertices correspond to the fragment's formulas, and the diagram's central symmetry corresponds to the fragment's PCD-structure) ${ }^{5}$ Each such polygon/polyhedron $\mathcal{P}$ has a number of reflectional and rotational symmetries, which constitute a group under the composition operation. This group is the symmetry group of $\mathcal{P}$ and will be denoted $\mathcal{S}_{\mathcal{P}}$ [28, p. 67]. Its cardinality $\left|\mathcal{S}_{\mathcal{P}}\right|$ measures how symmetric $\mathcal{P}$ is, and is thus strictly based on the geometrical properties of the polygon/polyhedron.

We now turn to the interaction between the numbers $2^{n} \times n$ ! and $\left|\mathcal{S}_{\mathcal{P}}\right|$. First of all, it should be noted that the former is typically larger than the latter, since every symmetry of $\mathcal{P}$ can also be seen as the result of permuting and changing the orientation of the PCDs that are visualized by $\mathcal{P}$, but not vice versa. Note, for example, that the hexagon in Fig. 3(a) can be seen as the result of reflecting the hexagon in Fig. 2(a) around the axis defined by $\square p$ and $\diamond \neg p$, but it can equally validly be seen as the result of permuting the PCDs $(\diamond p, \square \neg p)$ and $(\square p \vee \square \neg p, \diamond p \wedge \diamond \neg p)$ in the latter hexagon. By contrast, note that the hexagon in Fig. 3(b) can be seen as the result of changing the orientation of the PCD

\footnotetext{
${ }^{3}$ One counterexample is Chow [7], who studies Aristotelian diagrams that satisfy the logical condition, but not the geometrical condition.

${ }^{4}$ In [20, p. 77] this formula is applied to a fragment of 4 formulas (so $n=2$ ).

${ }^{5}$ In this paper, we will mainly focus on regular polygons and polyhedra (the only exception being the brief discussion of rectangles in Section 4). However, this restriction is only made for reasons of space; in principle, the account presented here can be applied to regular and non-regular shapes alike.
} 
$(\square p \vee \square \neg p, \diamond p \wedge \diamond \neg p)$ in the hexagon in Fig. 2(a), but that it is not the result of applying any reflection or rotation to the latter hexagon.

The key idea is now that the $2^{n} \times n$ ! different ways of ordering $n$ PCDs can be partitioned based on whether they yield variants of $\mathcal{P}$ that can be obtained from each other via reflections or rotations ${ }^{6}$ This partition has $\frac{2^{n} \times n !}{\left|\mathcal{S}_{\mathcal{P}}\right|}$ cells, which will be called fundamental forms. It follows immediately that diagrams with different fundamental forms are not reflectional or rotational variants of each other; and each fundamental form yields exactly $\left|\mathcal{S}_{\mathcal{P}}\right|$ diagrams that are all reflectional or rotational variants of each other. For example, the hexagons in Figs. 2(a) and 3(a) have the same fundamental form, whereas the hexagons in Figs. 2(a) and 3(b) have different fundamental forms.

Suppose now that we have two distinct polygons/polyhedra $\mathcal{P}$ and $\mathcal{P}^{\prime}$ that visualize the same $2 n$-formula fragment $\mathcal{F}$. Suppose, furthermore, that $\mathcal{P}$ is less symmetric than $\mathcal{P}^{\prime}$. This means that $\left|\mathcal{S}_{\mathcal{P}}\right|<\left|\mathcal{S}_{\mathcal{P}^{\prime}}\right|$, and hence $\left.\frac{2^{n} \times n !}{\left|\mathcal{S}_{\mathcal{P}}\right|}>\frac{2^{n} \times n !}{\left|\mathcal{S}_{\mathcal{P}^{\prime}}\right|}\right]$ i.e. $\mathcal{P}$ has more fundamental forms than $\mathcal{P}^{\prime}$. In other words, by having fewer symmetries, $\mathcal{P}$ makes a number of visual distinctions that are not made by $\mathcal{P}^{\prime}$. The quality of $\mathcal{P}$ and $\mathcal{P}^{\prime}$ as Aristotelian diagrams for the fragment $\mathcal{F}$ depends on whether these visual distinctions correspond to any logical distinctions in $\mathcal{F}$. On the one hand, if there are such logical distinctions present in $\mathcal{F}$, then $\mathcal{P}$ is to be preferred over $\mathcal{P}^{\prime}$, since $\mathcal{P}$ allows us to visualize these logical distinctions by mapping them onto the visual distinctions of its fundamental forms, whereas $\mathcal{P}^{\prime}$ would simply force us to collapse them. On the other hand, if there are no such logical distinctions present in $\mathcal{F}$, then $\mathcal{P}^{\prime}$ is to be preferred over $\mathcal{P}$, since in this case, the visual distinctions between the fundamental forms of $\mathcal{P}$ do not correspond to any logical differences in $\mathcal{F}$, but are merely by-products of the lack of symmetry in $\mathcal{P} 8$

\section{Aristotelian Diagrams with Two PCDs}

We will now apply the logico-geometrical account presented in the previous section to Aristotelian diagrams for fragments consisting of 2 PCDs. On the logical

\footnotetext{
${ }^{6}$ The idea of working up to symmetry can already be found in [4, p. 315], where it is stated that Aristotelian squares that are symmetrical variants of each other should be "counted as being of the same type". The assumed irrelevance of symmetry considerations for diagram design is also in line with work on other types of diagrams, such as Euler diagrams [27]: several of their visual characteristics have been investigated [13], but it has been found that rotation has no significant influence on user comprehension of Euler diagrams 2 .

7 Note that both fractions have the same numerator (since the two Aristotelian diagrams have the same logical properties, viz. they both visualize the fragment $\mathcal{F}$ ), but different denominators (since the two diagrams have different geometrical properties, viz. $\mathcal{P}$ is less symmetric than $\left.\mathcal{P}^{\prime}\right)$.

8 These considerations can be viewed as an application of the congruity/isomorphism principle in diagram design [18|36]: the visual properties of the diagram should closely correspond to the logical properties of the visualized fragment.
} 
side, one can show that the 2-PCD Aristotelian diagrams can be classified into exactly 2 Aristotelian families, viz. classical and degenerated 32 33. On the geometrical side, visualizing such 2-PCD diagrams requires a polygon/polyhedron that has 4 vertices and is centrally symmetric. In this section, we will focus on two such polygons, viz. the square and the rectangle (also recall Footnote 5).

Logically speaking, a 2-PCD fragment can be ordered in $2^{2} \times 2 !=8$ distinct ways. Geometrically speaking, the symmetry group $\mathcal{S}_{s q}$ of a square has order 8 , whereas the symmetry group $\mathcal{S}_{\text {rect }}$ of a proper (i.e. non-square) rectangle has order 4 . This difference reflects the fact that a square is a more symmetrical shape than a (proper) rectangle, since a rectangle distinguishes between its long and short edges, whereas the square collapses this distinction (by having 4 edges of the same length). Consequently, when a 2-PCD fragment is visualized by means of a square, this yields $\frac{2^{2} \times 2 !}{\left|\mathcal{S}_{s q}\right|}=\frac{8}{8}=1$ fundamental form; by contrast, when it is visualized by means of a rectangle, this yields $\frac{2^{2} \times 2 !}{\left|\mathcal{S}_{\text {rect }}\right|}=\frac{8}{4}=2$ fundamental forms.

Because it is less symmetrical, the rectangle makes more visual distinctions (long/short edges) than the square. In order to determine which shape is the most effective visualization of a 2-PCD fragment, we should investigate whether these visual distinctions correspond to any logical distinctions in the fragment. We will now do this for each of the two Aristotelian families of 2-PCD fragments.

Classical 2-PCD Fragments. Visualizing a classical 2-PCD fragment using a square yields 1 fundamental form; see e.g. Fig. 11(b). This means that all oriented permutations of the 2 PCDs yield diagrams that are rotational or reflectional variants of each other, regardless of where the (sub)contrarieties and subalternations are in the diagram. By contrast, visualizing this fragment by means of a (proper) rectangle yields 2 fundamental forms, as shown in Fig. 4 $4(a-b)$. In the first fundamental form, the (sub)contrarieties occupy the rectangle's long edges and the subalternations occupy its short edges, whereas in the second fundamental form it is the other way around.

Some authors have claimed that there is an important logical difference between the Aristotelian relations of (sub)contrariety on the one hand, and subalternation on the other. They distinguish between two complementary perspectives on the classical squar ${ }^{9}$ of opposition: as a theory of negation and as a theory of logical consequence [33. The former focuses on (sub)contrariety, while the latter focuses on subalternation. Furthermore, it has been argued that these two perspectives are linked to different scholarly traditions of Aristotle's logical works: the former is mainly found in commentaries on De Interpretatione, whereas the latter is central in commentaries on the Prior Analytics [8].

If a classical 2-PCD fragment is visualized by means of a rectangle, this logical distinction can directly be visualized, by putting the (sub)contrarieties and subalternations on edges of different lengths. For example, if one primarily focuses on the theory of negation, then one can put the (sub)contrarieties on the long edges, thus giving them more visual prominence [37, p. 515-516], while if

\footnotetext{
${ }^{9}$ We are using the term 'square' in a strictly historical sense here, regardless of its concrete geometrical properties.
} 

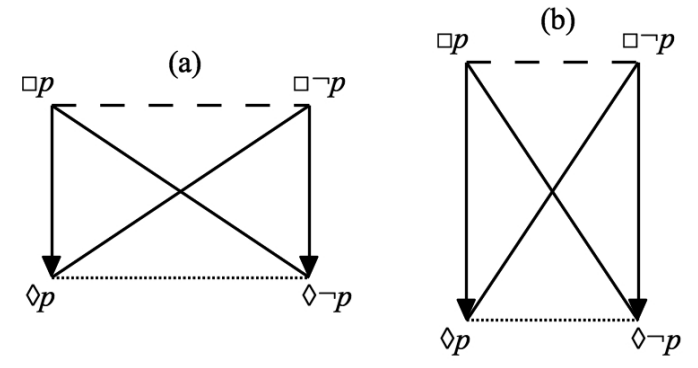

Fig. 4. The two fundamental forms of a (proper) rectangle for the classical 2-PCD fragment of S5-formulas that was already visualized by means of a square in Fig. 1(b).

one's focus is on the theory of consequence, one should rather put the subalternations on the long edges. By contrast, if the fragment is visualized using a square, then the distinction between (sub)contrariety and subalternation cannot be visualized in this way, since the square's edges are all of the same length.

Next to those who focus on the differences between (sub)contrariety and subalternation, there are also authors who rather emphasize the logical unity of these relations. They point out, for example, that every (sub)contrariety gives rise to two subalternations, and every subalternation gives rise to a contrariety and a subcontrariety [15/33, ${ }^{10}$ This has important consequences for the optimal visualization of a classical 2-PCD fragment. If the fragment is visualized by means of a square, then the unity of (sub)contrariety and subalternation is visualized by putting them all on edges of the same length. By contrast, if the fragment is visualized by means of a rectangle, then one will be forced to put either the (sub)contrarieties or the subaltnerations on the rectangle's long edges; however, this visual difference is not motivated by any logical considerations, but is merely a by-product of the lack of symmetry in the rectangle.

In sum: whether a square or a rectangle is the most suitable diagram for visualizing a classical 2-PCD fragment depends on one's logical views. If one focuses on the differences between the Aristotelian relations of (sub)contrariety and subalternation, then the rectangle is the optimal diagram, but if one rather focuses on the unity between those relations, then the square seems most suitable.

Degenerated 2-PCD Fragments. The formulas in a degenerated 2-PCD fragment are all pairwise unconnected (except for the two pairs of contradictory formulas, of course). Because of the strictly negative characterization of unconnectedness (absence of all Aristotelian relations), there do not seem to be any logical grounds for further differentiating between these pairs of unconnected formulas. Consequently, if the fragment is visualized by means of a square, then the

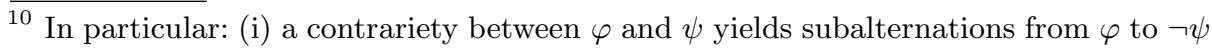
and from $\psi$ to $\neg \varphi$; (ii) a subcontrariety between $\varphi$ and $\psi$ yields subalternations from $\neg \varphi$ to $\psi$ and from $\neg \psi$ to $\varphi$; (iii) a subalternation from $\varphi$ to $\psi$ yields a contrariety between $\varphi$ and $\neg \psi$ and a subcontrariety between $\neg \varphi$ and $\psi$.
} 
equal logical status of the four pairs of unconnected formulas is visually represented by the fact that they are all on edges of the same length; see e.g. Fig. 11(c). By contrast, if one were to visualize the fragment using a rectangle, then one would be forced to choose two unconnected pairs to put on the rectangle's long edges, without having any logical motivation for doing so. The optimal diagram for visualizing a degenerated 2-PCD fragment thus seems to be a square, rather than a rectangle.

\section{$5 \quad$ Aristotelian Diagrams with Three PCDs}

In this section we continue our exploration of the logico-geometrical account presented in Section 3, by applying it to Aristotelian diagrams for fragments consisting of 3 PCDs. On the logical side, one can show that the 3-PCD Aristotelian diagrams can be classified into exactly 5 Aristotelian families, viz. JSB, $S C, U 4, U 8$ and U12 3133. On the geometrical side, visualizing such 3-PCD diagrams requires a centrally symmetric polygon/polyhedron with 6 vertices. We will consider two such shapes, viz. the hexagon (2D) and the octahedron (3D).

Logically speaking, a 3 -PCD fragment can be ordered in $2^{3} \times 3 !=48$ distinct ways. Geometrically speaking, the symmetry group $\mathcal{S}_{\text {hex }}$ of a hexagon has order 12 , whereas the symmetry group $\mathcal{S}_{\text {oct }}$ of an octahedron has order 48 . This difference reflects the fact that an octahedron is higher-dimensional than a hexagon, which allows it to have more symmetries (viz. one additional rotation axis). Consequently, when a 3-PCD fragment is visualized by means of a hexagon, this yields $\frac{2^{3} \times 3 !}{\left|\mathcal{S}_{\text {hex }}\right|}=\frac{48}{12}=4$ fundamental forms; by contrast, when it is visualized by means of an octahedron, this yields $\frac{2^{3} \times 3 !}{\left|\mathcal{S}_{o c t}\right|}=\frac{48}{48}=1$ fundamental form.

Because it is less symmetrical, the hexagon makes more visual distinctions than the octahedron. In order to determine which shape is the most effective visualization of a 3-PCD fragment, we should investigate whether these visual distinctions correspond to any logical distinctions in the fragment. This is exactly what we will do next, for each of the 5 Aristotelian families of 3-PCD fragments ${ }^{11}$

JSB 3-PCD Fragments. Visualizing a JSB fragment by means of an octahedron yields 1 fundamental form; see e.g. Fig. 3(c). By contrast, visualizing it by means of a hexagon yields 4 fundamental forms; see e.g. Figs. 2(a) and $5(a-c)$. In the first fundamental form, the 3 lines connecting the contrary formulas are all equally long, and thus constitute an equilateral triangle. In the other three fundamental forms, one line of contrariety is longer than the other two, yielding a (proper) isosceles triangle.

If the JSB fragment being visualized is (isomorphic to) the Boolean algebra $\mathbb{B}_{3}$ (except for its $T$ - and $\perp$-elements), then its 3 pairwise contrary formulas (in our S5-example: $\square p, \diamond p \wedge \diamond \neg p$ and $\square \neg p$ ) are all of the same level: their canonical bitstring representations are 100, 010 and 001 [14. Consequently, the

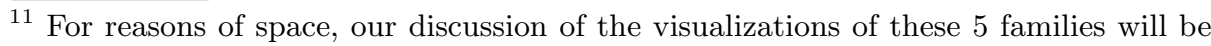
fairly brief; however, much more can (and should) be said about each of them. 

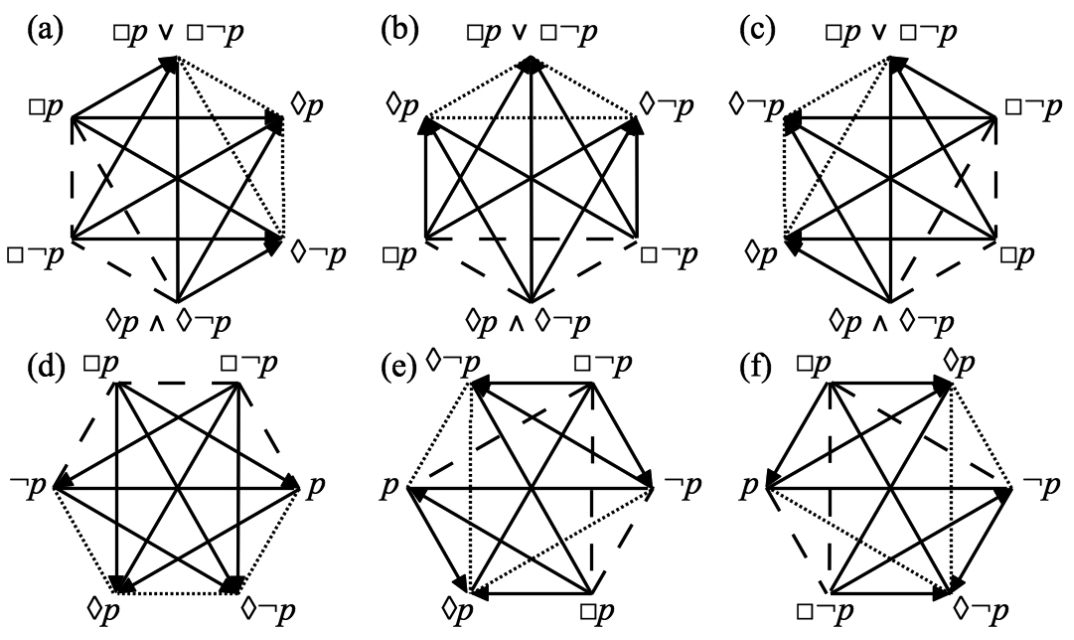

Fig. 5. (a-c) The three remaining fundamental forms of a hexagon for the JSB fragment of S5-formulas whose first fundamental form was already shown in Fig. 2(a); (d-f) the three remaining fundamental forms of a hexagon for the SC fragment of S5-formulas whose first fundamental form was already shown in Fig. 2(b).

3 contrarieties holding between them are all equally 'strong', and are thus best visualized using 3 lines of equal length [18/36, as in the hexagon in Fig. 2(a).

However, for linguistic-cognitive reasons it is sometimes useful to view a JSB fragment as (isomorphic to) a fragment of a much larger Boolean algebra, e.g. $\mathbb{B}_{5}$, since this allows us to treat the 3 pairwise contrary formulas as belonging to different levels. For example, in our S5-example, it makes sense to treat $\square p$ and $\square \neg p$ as the level-1 bitstrings 10000 and 00001, resp., since these formulas represent the two 'extremes' of a 'modal scale', while $\diamond p \wedge \diamond \neg p$ is treated as the level-3 bitstring 01110, since it represents the entire 'interior' of that modal scale 34. Consequently, the contrarieties holding between these formulas are of different 'strengths': the extremes $\square p$ and $\square \neg p$ are much more contrary to each other than they are to the intermediate $\diamond p \wedge \diamond \neg p$. It therefore makes sense to visualize the strongest contrariety (between $\square p$ and $\square \neg p$ ) by means of a line that is longer than the lines representing the two other contrarieties. This is exactly the case with the contrariety triangle in the hexagon in Fig. 5(b).

In sum: if the JSB fragment is visualized by means of a hexagon, then various logical distinctions can directly be visualized by using different fundamental forms for different cases. By contrast, if the fragment is visualized using an octahedron, then these distinctions are collapsed, since the octahedron has just a single fundamental form. The optimal diagram for visualizing a JSB fragment is thus a hexagon, rather than an octahedron 12

$\overline{12}$ This result is in line with earlier work on visualizations for JSB fragments [13 16]. 
SC 3-PCD Fragments. Visualizing an SC fragment by means of a hexagon yields 4 fundamental forms; see e.g. Figs. 2 (b) and 5 (d-f) ${ }^{13}$ In the two hexagons in Fig. 5(e-f), the subalternations do not share one common direction; in other words, the idea that the formulas are ordered (according to the strict partial order of subalternation) is not at all visualized in these hexagons. Furthermore, the (sub)contrarieties are visually most prominent: most of them are in the center of the diagram (the subalternations are in the periphery) and they are also longer than most of the subalternations 37. Turning to the hexagon in Fig. 5(d), we see that the subalternations do share a common direction (they all go downward), and hence, this diagram directly visualizes the ordering induced by the subalternations (lower in the diagram corresponds to further in the subalternation ordering). Furthermore, in this hexagon the subalternations are visually most prominent: they are all in the center of the diagram, and they are longer than all the (sub)contrarieties. Finally, the hexagon in Fig. 2(b) also directly visualizes the ordering induced by the subalternations (again: lower in the diagram corresponds to further in the subalternation ordering). In this hexagon, however, the (sub)contrarieties are visually most prominent: most of them are in the diagram's center, and they are longer than most of the subalternations.

Putting everything together, we thus find that the two hexagons in Fig. $5(\mathrm{e}-\mathrm{f})$ primarily draw the user's attention to the (sub)contrarieties in the SC fragment, and in order to achieve this, they even distort the ordering induced by the subalternations [36, p. 37]. Next, the hexagon in Fig. 5(d) focuses on the fragment's subalternation structure, by making the subalternations visually most prominent and also respecting the ordering induced by these subalternations. Finally, the hexagon in Fig. 2(b) strikes an ideal balance between these two extremes: it primarily draws the user's attention to the (sub)contrarieties in the SC fragment, but does so while still respecting the ordering induced by the subalternations.

Hence, if the SC fragment is visualized by means of a hexagon, then different fundamental forms can be used to visually emphasize different logical aspects of the fragment. By contrast, if one were to visualize the fragment by means of an octahedron, then this would no longer be possible, since the octahedron has just a single fundamental form. Consquently, the best diagram for visualizing a SC fragment seems to be a hexagon, rather than an octahedron.

U4 3-PCD Fragments. Visualizing a U4 fragment by means of a hexagon yields 4 fundamental forms; see e.g. Figs. 2(c) and 6(a-c). In Fig. 2(c), the (sub)contrarieties are visually most prominent: they are in the center of the diagram and they are also longer than the subalternations. In Fig. 6(b), it is exactly the other way around: the subalternations are in the center of the diagram, and they are longer than the (sub)contrarieties ${ }^{14}$ The hexagons in Figs. 2(c) and 6 (b) thus draw the user's attention to either the (sub)contrarieties or the subalternations. Recalling the logical importance of the distinction between these two

$\overline{{ }^{13} \text { Hexagons } 1,} 3$ and 6 in [6, p. 131-132] visualize an SC fragment using three distinct fundamental forms, viz. those shown in Figs. 2(b), 5 (e) and 5(f), respectively.

${ }^{14}$ The hexagons in Figs. 6(a) and 6.(c) strike a balance between the (sub)contrarieties and subalternations, by distributing visual prominence equally among them. 


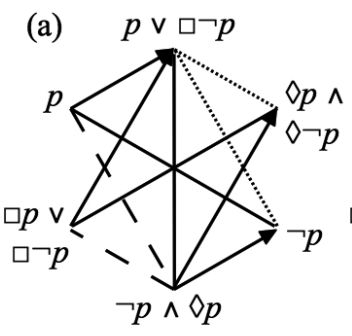

(d)

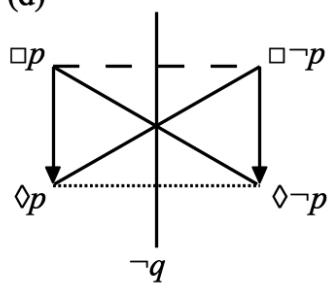

(b)

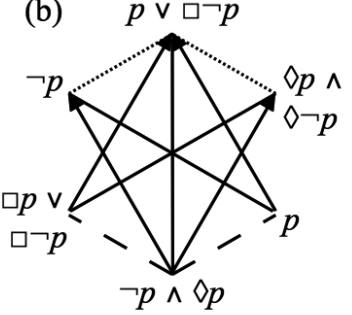

(e)

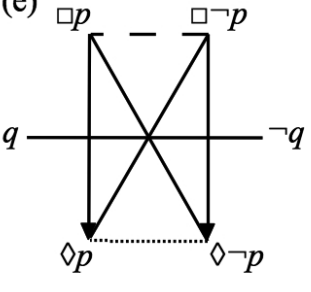

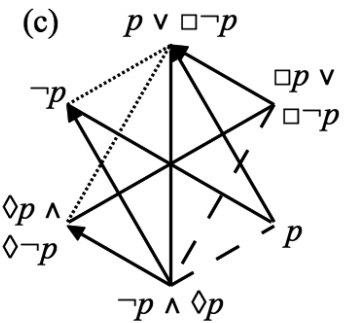

(f)

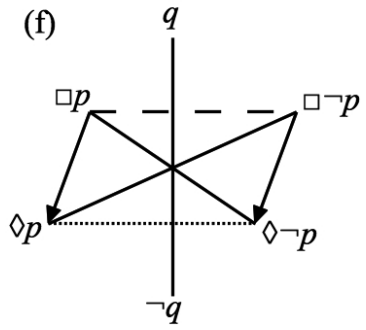

Fig. 6. $(\mathrm{a}-\mathrm{c})$ The three remaining fundamental forms of a hexagon for the U4 fragment of S5-formulas whose first fundamental form was already shown in Fig. 2(c); (d-e) two of the four fundamental forms of a hexagon for the U8 fragment $\{\square p, \square \neg p, \diamond p, \diamond \neg p, q, \neg q\}$; (f) (the unique fundamental form of) an octahedron for the same fragment.

types of Aristotelian relations (cf. Section 44), these two hexagons will thus be particularly useful, depending on the author's concrete purposes: does she want her audience to focus on the (sub)contrarieties or rather on the subalternations?

In sum: if the U4 fragment is visualized by means of a hexagon, then different fundamental forms can be used to visually emphasize different logical relations inside the fragment. By contrast, if one were to visualize the fragment by means of an octahedron, then this would no longer be possible, since the octahedron has just a single fundamental form. The optimal diagram for visualizing a U4 fragment is thus a hexagon, rather than an octahedron.

U8 3-PCD Fragments. A U8 fragment consists of four formulas that constitute a classical 2-PCD fragment, together with an additional pair of contradictory formulas that are unconnected to the first four. A typical example is the S5-fragment $\{\square p, \square \neg p, \diamond p, \diamond \neg p, q, \neg q\}$. Visualizing a U8 fragment by means of a hexagon yields 4 fundamental forms, viz. 2 fundamental forms in which the additional PCD is parallel to the subalternations, as in Fig. 6(d), and 2 fundamental forms in which the additional PCD is parallel to the (sub)contrarieties, as in Fig. 6(e). Since there does not seem to be any logical reason for preferring one option over the other, the differences between the 4 fundamental forms are thus mere side-products of the hexagon's lack of symmetry. By contrast, if one visualizes the fragment using an octahedron, then one can put the additional PCD perpendicular to the subalternations as well as the (sub)contrarieties (thereby avoiding any unmotivated design decisions), as in Fig. 6(f). In sum, then, the best diagram for visualizing a U8 fragment seems to be an octahedron. 
U12 3-PCD Fragments. The U12 fragments are the perfect analogues of the degenerated 2-PCD fragments, in the sense that their formulas are all pairwise unconnected (except for the 3 pairs of contradictory formulas, of course). A typical example is the CPL-fragment $\{p, \neg p, q, \neg q, r, \neg r\}$. If such a fragment is visualized by means of an octahedron, then the equal logical status of its 12 pairs of unconnected formulas is visually represented by the fact that they are all on lines of equal length (viz. the 12 edges of the octahedron). By contrast, if one were to visualize the fragment using a hexagon, one would be forced to put these unconnected pairs on lines of different lengths, without any logical motivation. The optimal diagram for visualizing a U12 fragment is thus an octahedron, rather than a hexagon.

\section{Conclusions and Future Work}

In this paper we have presented a systematic approach for dealing with informationally equivalent Aristotelian diagrams. The account is based on the interaction between the logical properties of the visualized fragment and the geometrical properties of the concrete polygon/polyhedron. Applying this account to all Aristotelian families of 2-PCD and 3-PCD fragments has led to several new insights: as to the 2-PCD fragments, the classical ones are best visualized by means of a rectangle if one focuses on the distinction between (sub)contrariety and subalternation and by means of a square otherwise, and the degenerated ones by means of a square; as to the 3-PCD fragments, JSB, SC and U4 are best visualized using a hexagon, and U8 and U12 using an octahedron.

A natural next step involves applying the account to 4-PCD fragments. This is by no means trivial, since there exist 18 Aristotelian families of 4-PCD fragments, only a few of which are currently well-understood. As for the geometric shapes to be used, obvious candidates include the (regular) octagon and the cube, which have symmetry groups of order resp. 16 and 48, and thus yield resp. $\frac{2^{4} \times 4 !}{16}=\frac{384}{16}=24$ and $\frac{2^{4} \times 4 !}{48}=\frac{384}{48}=8$ fundamental forms. However, when dealing with Aristotelian families that do not have any relevant logical distinctions to be visualized, one might also want to consider shapes with a symmetry group of order 384, since these will yield exactly $\frac{384}{384}=1$ fundamental form.

On a more general level, when visualizing an $n$-PCD fragment, one might want to consider a polytop ${ }^{15}$ that is (i) centrally symmetric, (ii) has $2 n$ vertices, and (iii) has a symmetry group of order $2^{n} \times n$ ! (since such a polytope will yield exactly $\frac{2^{n} \times n !}{2^{n} \times n !}=1$ fundamental form). There indeed exists a polytope satisfying these criteria, for all $n$, viz. the cross-polytope of dimension $n$, which is the dual polytope of the $n$-dimensional hypercube [9, p. 121, 294]. In case $n=2$, this is the dual of a square, which is itself also a square (cf. Section 4); in case $n=3$, this is the dual of a cube, which is an octahedron (cf. Section 5 ).

The practical usefulness of these last observations is fairly limited, because they involve (cross-)polytopes of arbitrarily high dimensions, which are not very

${ }_{15}$ The term 'polytope' is a generalization of the terms 'polygon' and 'polyhedron' to arbitrary dimensions [9]. 
useful for concrete visual-diagrammatic purposes. For example, visualizing a 4PCD fragment would require a so-called 16-cell (i.e. the dual of the 4-dimensional hypercube) [9, p. 292], which can be studied abstractly, but goes beyond human visual cognition ${ }^{16}$ Nevertheless, the theoretical importance of these observations should not be underestimated, since they show that the diagrams that several logicians have come up with to visualize 2- and 3-PCD fragments 'up to symmetry' (i.e. having a unique fundamental form), viz. the square and the octahedron, are the first few instances of a well-defined, infinite series of polytopes.

Acknowledgements We would like to thank Dany Jaspers and Margaux Smets for their valuable feedback on an earlier version of this paper. The first author holds a Postdoctoral Fellowship of the Research Foundation-Flanders (FWO).

\section{References}

1. Benoy, F., Rodgers, P.: Evaluating the comprehension of Euler diagrams. In: 11th Int. Conf. Information Visualization, pp. 771-778. IEEE Computer Society (2007)

2. Blake, A., Stapleton, G., Rodgers, P., Cheek, L., Howse, J.: Does the orientation of an Euler diagram affect user comprehension? In: 18th Int. Conf. on Distributed Multimedia Systems, pp. 185-190. Knowledge Systems Institute (2012)

3. Blake, A., Stapleton, G., Rodgers, P., Cheek, L., Howse, J.: The impact of shape on the perception of Euler diagrams. In: Dwyer, T., Purchase, H., Delaney, A. (eds.) Diagrams 2014, pp. 123-137. LNCS 8578, Springer (2014)

4. Brown, M.: Generalized quantifiers and the square of opposition. Notre Dame Journal of Formal Logic 25, 303-322 (1984)

5. Cavaliere, F.: Fuzzy syllogisms, numerical square, triangles of contraries, interbivalence. In: Béziau, J.Y., Jacquette, D. (eds.) Around and Beyond the Square of Opposition, pp. 241-260. Springer (2012)

6. Chatti, S., Schang, F.: The cube, the square and the problem of existential import. History and Philosophy of Logic 32, 101-132 (2013)

7. Chow, K.F.: General patterns of opposition squares and 2n-gons. In: Béziau, J.Y., Jacquette, D. (eds.) Around and Beyond the Square, pp. 263-275. Springer (2012)

8. Correia, M.: Boethius on the square of opposition. In: Béziau, J.Y., Jacquette, D. (eds.) Around and Beyond the Square of Opposition, pp. 41-52. Springer (2012)

9. Coxeter, H.S.M.: Regular Polytopes. Dover Publications (1973)

10. Demey, L.: Algebraic aspects of duality diagrams. In: Cox, P.T., Plimmer, B., Rodgers, P. (eds.) Diagrams 2012, pp. 300-302. LNCS 7352, Springer (2012)

11. Demey, L.: Structures of oppositions for public announcement logic. In: Béziau, J.Y., Jacquette, D. (eds.) Around and Beyond the Square of Opposition, pp. 313339. Springer (2012)

\footnotetext{
${ }^{16}$ One might object that higher-dimensional cross-polytopes can be visualized, by considering their lower-dimensional projections. This objection fails to take into account, however, that such projections will break some of the cross-polytopes' symmetries, and thus not be useful for our current purposes. This phenomenon can already be observed in very low dimensions; for example, the hexagon in Fig. 2(a) can be seen as the $2 \mathrm{D}$ projection of the octahedron in Fig. 3(c), but this projection drastically reduces the number of symmetries (from the octahedron's 48 to the hexagon's 12).
} 
12. Demey, L.: Interactively illustrating the context-sensitivity of Aristotelian diagrams. In: Christiansen, H., Stojanovic, I., Papadopoulos, G. (eds.) Modeling and Using Context, pp. 331-345. LNCS 9405, Springer (2015)

13. Demey, L., Smessaert, H.: The relationship between Aristotelian and Hasse diagrams. In: Dwyer, T., Purchase, H., Delaney, A. (eds.) Diagrams 2014, pp. 213-227. LNCS 8578, Springer (2014)

14. Demey, L., Smessaert, H.: Combinatorial bitstring semantics for arbitrary logical fragments. Submitted (2015)

15. Demey, L., Smessaert, H.: Metalogical decorations of logical diagrams. Logica Universalis (Forthcoming)

16. Demey, L., Smessaert, H.: Shape heuristics in Aristotelian diagrams. In: Kutz, O., Borgo, S., Bhatt, M. (eds.) Shapes 3.0 Proceedings. CEUR-WS (Forthcoming)

17. Dubois, D., Prade, H.: From Blanché's hexagonal organization of concepts to formal concept analysis and possibility theory. Logica Universalis 6, 149-169 (2012)

18. Gurr, C.: Effective diagrammatic communication: Syntactic, semantic and pragmatic issues. Journal of Visual Languages and Computing 10, 317-342 (1999)

19. Horn, L.R.: A Natural History of Negation. University of Chicago Press (1989)

20. Jacquette, D.: Thinking outside the square of opposition box. In: Béziau, J.Y., Jacquette, D. (eds.) Around and Beyond the Square, pp. 73-92. Springer (2012)

21. Joerden, J.: Logik im Recht. Springer (2010)

22. Kraszewski, Z.: Logika stosunków zakresowych [Logic of extensional relations]. Studia Logica 4, 63-116 (1956)

23. Larkin, J., Simon, H.: Why a diagram is (sometimes) worth ten thousand words. Cognitive Science 11, 65-99 (1987)

24. McNamara, P.: Deontic logic. In: Zalta, E.N. (ed.) Stanford Encyclopedia of Philosophy. CSLI (2010)

25. Moretti, A.: The geometry of standard deontic logic. Log. Univers. 3, 19-57 (2009)

26. Parsons, T.: The traditional square of opposition. In: Zalta, E.N. (ed.) Stanford Encyclopedia of Philosophy. CSLI (2006)

27. Rodgers, P.: A survey of Euler diagrams. Journal of Visual Languages and Computing 25, 134-155 (2014)

28. Rotman, J.J.: An Introduction to the Theory of Groups (4th ed.). Springer (1995)

29. Seuren, P., Jaspers, D.: Logico-cognitive structure in the lexicon. Language 90, 607-643 (2014)

30. Smessaert, H.: On the 3D visualisation of logical relations. Logica Universalis 3, 303-332 (2009)

31. Smessaert, H.: Boolean differences between two hexagonal extensions of the logical square of oppositions. In: Cox, P.T., Plimmer, B., Rodgers, P. (eds.) Diagrams 2012, pp. 193-199. LNCS 7352, Springer (2012)

32. Smessaert, H., Demey, L.: Logical and geometrical complementarities between Aristotelian diagrams. In: Dwyer, T., Purchase, H., Delaney, A. (eds.) Diagrams 2014, pp. 246-260. LNCS 8578, Springer (2014)

33. Smessaert, H., Demey, L.: Logical geometries and information in the square of opposition. Journal of Logic, Language and Information 23, 527-565 (2014)

34. Smessaert, H., Demey, L.: The unreasonable effectiveness of bitstrings in logical geometry. Submitted (2015)

35. Smessaert, H., Demey, L.: Visualising the Boolean algebra $\mathbb{B}_{4}$ in 3D. In: Jamnik, M., Uesaka, Y. (eds.) Diagrams 2016. LNCS, Springer (2016)

36. Tversky, B.: Prolegomenon to scientific visualizations. In: Gilbert, J.K. (ed.) Visualization in Science Education, pp. 29-42. Springer (2005)

37. Tversky, B.: Visualizing thought. Topics in Cognitive Science 3, 499-535 (2011) 